\title{
Correction to: Comparison of three different internal fixation implants in treatment of femoral neck fracture-a finite element analysis
}

Jia $\mathrm{Li}^{1+}$, Zhe Zhao ${ }^{2+}$, Pengbin Yin ${ }^{1}$, Licheng Zhang ${ }^{1 *}$ and Peifu Tang ${ }^{1 *}$

\section{Correction to: J Orthop Surg Res \\ https://doi.org/10.1186/s13018-019-1097-x}

In the original publication of this article [1], the word "place" should be changed to "plate". This word occurs at four sections: 1 . Methods of the Abstract; 2. Keywords of the Abstract; 3 Introduction; 4. Abbreviations.

\section{Author details}

'Department of Orthopedics, Chinese PLA General Hospital, No. 28 Fuxing Road, Beijing 100853, People's Republic of China. ${ }^{2}$ Department of

Orthopaedics, Beijing Tsinghua Changgung Hospital, School of Clinical Medicine, Tsinghua University, No. 168, Li Tang Road, Changping District, Beijing 102218, China.

Published online: 16 April 2019

\section{Reference}

1. Li, et al. Comparison of three different internal fixation implants in treatment of femoral neck fracture - a finite element analysis. J Orthop Surg Res. 2019;14:76 https://doi.org/10.1186/s13018-019-1097-x.

\footnotetext{
*Correspondence: zhanglcheng218@126.com; pftang301@163.com

†jia Li and Zhe Zhao contributed equally to this work.

'Department of Orthopedics, Chinese PLA General Hospital, No. 28 Fuxing

Road, Beijing 100853, People's Republic of China

Full list of author information is available at the end of the article
} 